\title{
Caracterización socio-económica de la cría de bovinos en la agricultura familiar del Alto Egipto
}

\author{
Molina-Flores, B. ${ }^{1}$, , Velasco, G. ${ }^{2}$, Camacho, M.E. ${ }^{3}$, Martínez, A.M. ${ }^{1}$ and Delgado, J.V. ${ }^{1}$
}

'Genetics Department. University of Cordoba. Cordoba. Spain.

${ }^{2}$ Genetic Resources Branch. FAO. Rome, Italy.

${ }^{3}$ Institute of Agriculture and Fisheries Research and Training (IFAPA). Córdoba. Spain.

\section{PALABRAS CLAVE ADICIONALES}

Comunidades rurales.

Factores socio-económicos.

Sistemas locales.

Recursos zoo-genéticos.

Baladi.

\section{RESUMEN}

El Egipto actual es una entidad compleja que enfrenta una lucha contra el crecimiento de la población y la pobreza en el camino hacia un desarrollo humano equitativo. Esto es particularmente cierto en el Alto Egipto, donde más de la mitad de la población rural se considera en situación de pobreza, siendo la agricultura familiar, y en particular la cría de bovinos, una de las principales fuentes de ingresos. Para identificar los factores socioeconómicos que influyen en los sistemas tradicionales de agricultura familiar dedicados a la cría de bovinos, así como evaluar los conocimientos, aptitudes y prácticas de los productores pecuarios del Alto Egipto, se diseñó un estudio basado en entrevistas individuales y colectivas realizadas en hogares seleccionados de las gobernaciones de Minya, Assiut, Sohag, Quena y Luxor. Los resultados muestran como la cría de bovinos en esta región se ve significativamente obstaculizada por el limitado acceso a la tierra, el alto costo y escasa disponibilidad de insumos básicos, la marcada estacionalidad de la productividad animal, unos servicios de extensión pecuaria insuficientes y la dificultad para acceder a créditos y préstamos. Además, las estrategias y programas de mejora genética nacionales han descuidado las razas bovinas autóctonas (Baladi), a pesar de ser consideradas por los productores más adaptadas y resistentes a las severas condiciones locales. La comprensión de estos factores es esencial para la formulación e implementación de estrategias de intervención destinadas a mejorar la calidad de vida de estas comunidades rurales, así como preservar y gestionar los recursos zoogenéticos bovinos que explotan.

\section{Socio-economic characterization of cattle breeding in Upper Egypt's family farming}

\section{SUMMARY}

\section{ADDITIONAL KEYWORDS}

\section{Rural communities.}

Socio-economic factors.

Local Systems.

Animal genetic resources.

Baladi.

\section{INFORMATION}

\section{Cronología del artículo.}

Recibido/Received: 19.11.2017

Aceptado/Accepted: 26.09.2018

On-line: 15.01 .2019

Correspondencia a los autores/Contact e-mail:

I82moflb@uco.es
Today's Egypt is a complex entity, which faces a fight against population growth and poverty on the road to equitable human development. This is particularly true in Upper Egypt, where more than half of the rural population is considered to be in a situation of poverty, being family farming, and particularly cattle breeding, one of the main sources of income. In order to identify the socioeconomic factors that influence the family farming traditional systems dedicated to cattle breeding, as well as to evaluate the knowledge, aptitudes and practices of the Upper Egypt's cattle producers, a study was designed based on individual and collective interviews within selected households from the governorates of Minya, Assiut, Sohag, Quena and Luxor. The results show how callle breeding in this region is significantly hampered by limited access to land, high cost and scarce availability of basic inputs, the marked seasonality of animal productivity, insufficient livestock extension services and difficut ties in accessing credits and loans. In addition, national breeding strategies and programs have neglected the indigenous bovine breeds (Baladi), despite being considered by producers more adapted and resistant to the severe local conditions. The understanding of these factors is essential for the formulation and implementation of intervention strategies aimed at improving the quality of life of these rural communities, as well as preserving and managing the bovine animal genetic resources that they exploit.

\section{INTRODUCCIÓN}

La República Árabe de Egipto tiene una superficie total de $1.001 .450 \mathrm{~km}^{2}$, pero tan solo el 3,6\% se consideran aptos para la agricultura, de los cuales casi dos terceras partes corresponden a las tierras bañadas por el río Nilo (CIA 2018). De forma simplificada, la zona habitable del país se puede dividir en dos regiones físicas principales: el Valle del Nilo (Alto Egipto) y el Delta del Nilo (Bajo Egipto). El Alto Egipto está formado administrativamente por siete gobernaciones: Beni Suef, Minya, Assiut, Sohag, Quena, Luxor y Asuán (MAEC 2018).

La alta densidad de población (1.153,3 habitantes/ $\mathrm{km}^{2}$ en la superficie habitada) y el alto crecimiento 
demográfico (2,4\% anual), son considerados como los más graves problemas del país (ECES 2017). La población rural en Egipto representa el 57,3\% de la población total del país, proporción que en el Alto Egipto alcanza el 72,8\% (CAPMAS 2017). Las proyecciones para el 2050 prevén un aumento en la población que alcanzará los 151,1 millones de personas, el 43,5\% de las cuales vivirán en áreas rurales (FAO 2017).

En el año 2015 el 27,8\% de la población egipcia se encontraba en situación de pobreza, con especial incidencia sobre la población rural y, en particular, las familias con escaso acceso a la propiedad de tierras. Estos índices están directamente relacionados con el tamaño familiar: el $44 \%$ de las familias con entre seis y siete miembros son pobres. La alta tasa de analfabetismo refleja como la educación es el principal factor determinante de la pobreza, en especial en las áreas rurales del país (50,6\%). Las gobernaciones de Beni Suef $(30,2 \%)$, Sohag $(30,0 \%)$, Minya $(27,9 \%)$ y Assiut (26,5\%) presentaban en 2016 los índices más altos de analfabetismo del país (CAPMAS 2017).

La agricultura es el sector productivo que más personas emplea en Egipto $(27,5 \%)$ y el mayor empleador de la mujer, resultando fundamental para la seguridad alimentaria de las comunidades rurales (MAEC 2018). Aunque la contribución de la agricultura a la economía nacional ha ido disminuyendo en el último lustro, el sector aportó casi 40 billones de dólares en el año fiscal 2015/16, lo que representa cerca del 12\% del producto interno bruto (PIB) (WBG 2018).

Desde el punto de vista agro-ecológico, el Alto Egipto (Valle del Nilo) se caracteriza por unos suelos de origen aluvial que van de arcillosos a limosos, temperaturas medias de $20^{\circ} \mathrm{C}$ en invierno y $43^{\circ} \mathrm{C}$ en verano,y precipitaciones prácticamente inexistentes (FAO 2005). La irrigación con aguas del Nilo permite practicar la rotación intensiva de cultivos, con un promedio de más de dos cosechas al año, que incluye diversos tipos de cereales, leguminosas, hortalizas, frutas, forrajes y otros cultivos (ENID 2011).

No obstante, el sector agrícola en Egipto es extremadamente sensible al cambio climático. Los expertos estiman que, el aumento previsto de la temperatura en 2050 provocará una disminución de hasta el 20\% en la productividad de los principales cultivos, afectando también negativamente a la producción pecuaria. Las medidas de adaptación para incrementar la capacidad de resistencia al cambio climático incluyen el uso de variedades vegetales y razas animales nativas, adaptadas a las características agroecológicas locales y más tolerantes a los efectos provocados por el cambio climático (IFAD 2015).

Según el censo agrícola nacional, hay cerca de 3 millones de pequeñas propiedades, el $96 \%$ de las cuales tienen entre 3 y 5 feddans de superficie $(1$ feddan = 0,42 hectáreas) (Lewis 2008). El Alto Egipto cuenta con 2,7 millones de feddans de área física cultivable (31\% de la superficie nacional), sin embargo, la tenencia total de tierras agrícolas en la región es de 2 millones de feddans $(30,5 \%$ del total nacional), con cerca de 1,8 millones de propietarios (42\% del total del país) (ENID 2011). Esta gran fragmentación de las tierras agrícolas del país, y del Alto Egipto en particular, tiene una implicación directa en los sistemas de producción agropecuaria tradicionales (Thomson et al.2000).

Aunque el 95\% de las explotaciones familiares crían animales, la ganadería se considera una actividad complementaria al cultivo agrícola (MALR 2006). No obstante, el valor monetario de la producción animal total en 2016 fue de 10,4 billones de \$US y la importancia relativa de la ganadería en la producción agrícola total fue del 41\%, contribuyendo al 5\% del PIB (FAO 2017, FAOSTAT 2018). El 23\% del valor de la producción pecuaria total proviene del sector bovino y bufalino, que contribuyen con 5,8 billones de US\$, de los cuales el $66 \%$ es generado por la producción de carne y el 34\% por la de leche (FAO 2018). No es posible encontrar información del papel de la mujer en cuanto a impacto económico (Torres et al., 2016).

A pesar de la importancia de la producción pecuaria en la economía agrícola, ésta no es suficiente para suplir la demanda nacional y Egipto se ve obligado a importar animales vivos y productos de origen animal. En 2016 el país importó el equivalente a 8,7 billones de $\$$ US en animales vivos y productos de origen animal, mientras que exportó 2,9 billones de \$US. El 86,0\% del valor de las importaciones totales de productos agropecuarios en Egipto (10 billones de \$US) corresponden a animales vivos y productos de origen animal, lo cual indica la baja productividad del sector (FAOSTAT 2018).

Sin embargo, existen otros aspectos de la producción pecuaria que, aunque no suelen ser considerados en las estadísticas nacionales, contribuyen al sector agrícola nacional. Debido al escaso nivel de mecanización de la agricultura familiar egipcia, el valor de la tracción animal y el estiércol para fertilizar los campos se estima en un $40 \%$ del valor total de la producción ganadera, representando una entrada directa a la producción de cultivos (El-Shimi \& Arafa 2011). Para realizar trabajos agrícolas, los productores familiares mantienen razas bovinas nativas (Baladi) de triple propósito (Fitch \& Soliman 1981).

En la agricultura familiar egipcia, el ganado constituye también una fuente flexible de dinero en efectivo, permitiendo comprar insumos agrícolas y cumplir con otras necesidades familiares. Como reportado en otras regiones (Rangel-Quinto et al.,2016), la cría de animales supone también una red de seguridad ante la variabilidad de los precios de los cultivos, las malas cosechas $\mathrm{u}$ otros eventos de riesgo (Tabana et al.,2000). Para los pequeños propietarios y los sin tierra, los pequenos rumiantes representan la principal estrategia para escapar de la pobreza, pero tan pronto como pueden invierten en bovinos o búfalos (Alary et al.,2015).

La agricultura familiar es predominante a nivel nacional y generan una cantidad considerable de alimentos de origen animal, tanto para uso doméstico como comercial, pero al tratarse en la mayoría de los casos, de un mercado informal, no suelen existir datos de producción. La carga ganadera viene determinada por el tamaño del área destinada a cultivos forrajeros de la explotación, variando entre 1 y 10 cabezas de ganado(MALR 2009). Estos sistemas albergan al 33\% 
de la población bovina nacional (4,9 millones de cabezas) (FAOSTAT 2018), un tercio de la cual se localiza en el Alto Egipto y son animales de raza local o Baladi $(68,6 \%)$ o sus cruces $(29,1 \%)$, lo que indica que los sistemas tradicionales de producción pecuaria son especialmente dominantes en esta región (Osman et al., 2017).

En su mayor parte, es un sistema mixto agro-ganadero de "corte y acarreo" en el que, por lo general, se dedica un tercio del área de la explotación para plantar forraje para el ganado que, junto con los residuos de los cultivos principales y otros subproductos de la industria agrícola, constituyen el grueso de la alimentación animal (Thomson et al.,2000). Por tanto, requiere pocos insumos, pero las producciones son escasas, debido a un manejo poco eficiente y a unos bajos índices reproductivos. Aunque el ganado es vacunado durante las campañas gubernamentales, la mayoría de los productores familiares rara vez acceden a otros servicios veterinarios (FAO 2018). Para diversificar el riesgo y maximizar los recursos disponibles, este sistema tradicional mantiene varias especies animales con diferentes funciones productivas (FAO 2017) (Figura 1).

La época de partos suele ser en invierno (octubremarzo), coincidiendo con la mayor producción de forrajes. Algunos productores suelen vender los terneros lo antes posible para ahorrar leche y obtener dinero rápido para comprar alimentos concentrados o cubrir el costo del arrendamiento de la tierra. Otros prefieren conservarlos para ceba y usan las vacas como nodrizas, pues la leche de las búfalas es más valorada económicamente. La decisión de optar por una u otra estrategia de comercialización puede variar de un año a otro según las necesidades (Radwan 2016).

Debido a la mayor oferta y menor demanda, en invierno el precio de la carne disminuye un $20-40 \%$ con respecto al verano. El $65-75 \%$ de la producción anual de leche se concentra en invierno y primavera, cuando el forraje verde está disponible. El 38\% de la producción total de leche se destina al consumo doméstico y los excedentes se comercializan en los mercados locales en forma de leche, queso y/o mantequilla, siendo utilizados los ingresos generados para cubrir las necesidades básicas diarias de la familia (Radwan 2016, Soliman \& Mashhour 2011).

En el Alto Egipto, varios factores socio-económicos y culturales determinan que las explotaciones familiares estén más orientadas hacia la producción y comercialización de carne mediante el cebo de animales, liberando al hogar de la necesidad de los ingresos derivados de la venta de leche. La tradición popular considera que la leche es un alimento infantil, no aptopara adultos, y que su venta es una vergüenza social. Además, el gran tamaño de las familias conlleva un mayor consumo doméstico de lácteos, reduciendo los

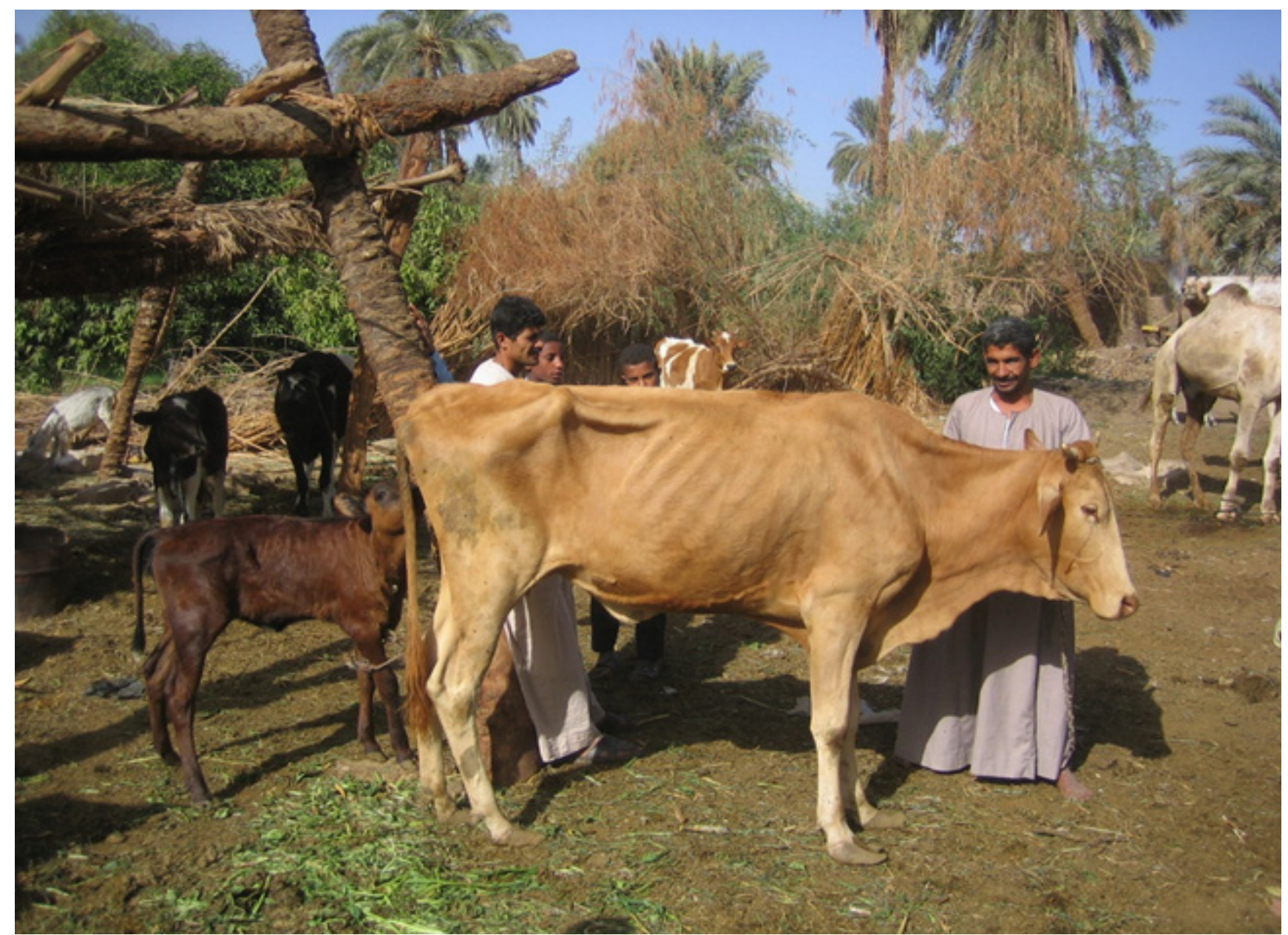

Figura 1. Sistema agrícola familiar de cría de bovinos en el Alto Egipto (Cattle breeding in Upper Egypt's family farming system) 
excedentes destinados para la venta (Soliman \& Mashhour 2011).

Muchas familias rurales no pueden criar ganado porque no tienen tierra propia, por tanto, no tienen espacio para alojar animales ni para producir forraje. Otras poseen explotaciones demasiado pequeñas que les impide mantener suficientes animales y, además, se ven obligados a comprar forrajes para alimentarlos. Así pues, los ingresos que se obtienen a través de la cría de ganado no son suficientes para mantener a las familias. Estos son factores cruciales que repercuten en el nivel de pobreza de las comunidades rurales de Egipto, pues es principalmente a través de los recursos adicionales que proporciona el ganado que pueden aumentar sus ingresos por encima del umbral de pobreza (IFAD 2015).

Además de estos factores, la producción pecuaria se ve significativamente obstaculizada por la baja productividad de la cabaña ganadera nacional. Mejoras en la alimentación, la salud, la genética y el manejo en la cría de bovinos, tendría una repercusión inmediata en las producciones pecuarias a nivel local y nacional (Fitch \& Soliman 1983). Además, facilitar la comercialización de productos de origen animal, así como el acceso a créditos y préstamos para la producción pecuaria, ayudaría también a mejorar las condiciones socio-económicas de la gran mayoría de los productores familiares (Lewis 2008).

El aumento en la demanda de productos de origen animal en los países en vías de desarrollo que viene produciéndose en las últimas décadas también está sucediendo en Egipto de manera proporcional al aumento de su población (Galal 2007). En 2050 la demanda nacional de leche y carne se estima en 23.888 mil y 5.401 mil toneladas respectivamente, lo que implica que la producción nacional deberá crecer a un ritmo anual de un 3,5\% para la leche y un $4,2 \%$ para la carne. Los sistemas de producción intensiva aumentarán su producción con relativa facilidad, pero los pequeños productores familiares se enfrentarán a grandes desafíos para mejorar su productividad (FAO 2017).

La "Estrategia de Desarrollo Agrícola Sostenible 2030" del gobierno egipcio ha marcado como objetivo principal la intensificación de la producción pecuaria, con el fin de satisfacer las demandas internas de proteínas de origen animal y reducir las importaciones al mínimo. A través del fomento de la producción avícola, se espera que el consumo de carne roja per cápita disminuya a una tasa de $0.5 \mathrm{~kg}$ cada cinco años y que la tasa de producción baje al 2\%. En el caso de la leche, se planea aumentar la producción hasta alcanzar un consumo medio per cápita de lácteos de 90 kg (MALR 2009).

Con este significativo crecimiento previsto en el sector ganadero egipcio, sería aconsejable planificar anticipadamente las acciones necesarias para garantizar así un desarrollo saludable y sostenible del sector en los próximos años, evitando así incrementar aún más el empobrecimiento rural.En Egipto, la diversidad zoogenética de los sistemas de producción tradicionales se está perdiendo y la intensificación de la producción animal está provocando un aumento de la demanda de agua y de la competencia por las tierras de uso agrícola, convirtiendo al ganado en una carga medioambiental en lugar de en un activo agroecológico (FAO 2017).

Por otra parte, el creciente reconocimiento del cambio climático está induciendo al país a promover cada vez más el desarrollo de una agricultura sostenible y esforzarse para mejorar la eficiencia y la inclusión de sus sistemas de producción pecuaria, de manera que puedan adaptarse a los efectos que puede producir este fenómeno (El-Ramady et al.,2013). Evaluar el impacto actual y a largo plazo de los sistemas de producción pecuaria en la economía y los medios de subsistencia de las comunidades rurales del país, identificando los desafíos que enfrentarán y las medidas de mitigación de riesgos necesarias, resulta fundamental para lograr un desarrollo económico y social integral, basado en un sector ganadero dinámico capaz de un crecimiento sostenido (FAO 2018).

Será necesario aplicar estrategias adecuadas para mejorar la producción de alimentos de origen animal en calidad y cantidad, las cuales deberán considerar los diferentes aspectos sociales y económicos de la producción ganadera, ser inclusivas e involucrar a todas las partes interesadas, garantizando la gestión sostenible de los recursos naturales y zoo-genéticos (FAO 2017). De esta forma, a través de la producción pecuaria y de la cría de bovinos en particular - considerando su importancia socio-económica, su posición prioritaria en el marco de las políticas nacionales actuales y su previsible crecimiento en las próximas décadas - Egipto logrará mejorar los medios de vida de los comunidades rurales e incluso, a algunos de los más desfavorecidos, salir de la pobreza (FAO 2018).

En este contexto, la razón principal del presente estudio es comprender cuáles son los factores socio-económicos que influyen en los sistemas tradicionales de agricultura familiar dedicados a la cría de bovinos, así como evaluar los conocimientos, aptitudes y prácticas de los productores pecuarios del Alto Egipto. El estudio se realizó en el marco del "Proyecto hispano-egipcio para el desarrollo de la inseminación artificial en el Alto Egipto", financiado por la Agencia Española de Cooperación Internacional y Desarrollo (AECID), el cual tenía por objeto el incremento de la seguridad alimentaria mediante la extensión y transferencia de tecnologías relacionadas con la ganadería a las comunidades rurales del Alto Egipto. El estudio fue planificado por el personal del proyecto junto con la contraparte nacional, y contó con el apoyo de una empresa de consultaría local para su ejecución a nivel de campo.

\section{MATERIAL Y MÉTODOS}

Con base a los resultados de una revisión de la literatura y las informaciones suministradas por los directores de los servicios veterinarios oficiales y los puntos focales del proyecto hispano-egipcio, tanto a nivel central en El Cairo, como en cada una de las gobernaciones objeto del estudio (Minya, Assiut, Sohag, Quena y Luxor), se elaboró un borrador de cuestionario para implementar una encuesta estructurada (Lewis-Beck et al.,2004). El mismo fue testado (mediante diez entrevistas individuales y dos entrevistas grupales a productores pecuarios en una localidad 


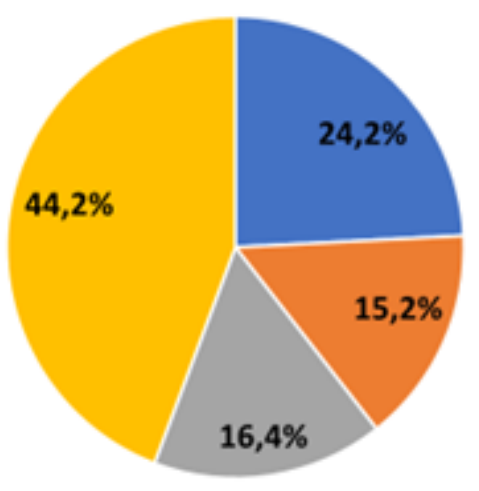

Área cultivada

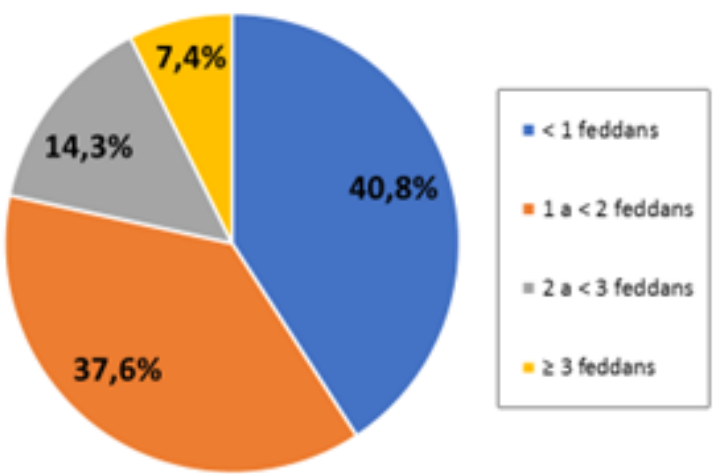

Cultivos forrajeros

Figura 2. Distribución de tierra cultivada y forrajes (Distribution of cultivated land and fodder crops).

rural de la gobernación de Giza), revisado y redactado en su formato final, garantizando el adecuado ingreso y posterior análisis de los datos obtenidos. A partir de la versión final del cuestionario se desarrollaron unas directrices para entrevistar otros grupos de productores familiares, que fueron utilizadas posteriormente en una investigación de evaluación rápida (Coghlan \& BrydonMiller 2014), con la intención de realizar una verificación cruzada de las respuestas obtenidas en la encuesta.

Para la recolección de datos, se seleccionaron un total de 30 municipios en base a su participación en el proyecto hispano-egipcio en las gobernaciones de Minya (Nazlet Hussein Ali, Qulosna, El-Beho, Abou Qorqas, El-Balad, El-Fekria, Derwa y Talaa), Assiut (El-Nawawra, El-Moutia, Doina, Baqoura, El-Berba, Awlad Elias y El-Dairout), Sohag (Dar El-Salam, Tahta, Meshta, Shatora, Awlad Ismail y El-Betakh), Quena (Quena, Abnoud, El-Waqf, El-Adaima y El-Dair) y Luxor (Luxor, El-Boghdadi, El-Odisat y ElQorana). Para la encuesta estructurada se seleccionó una muestra aleatoria de un total de 434 productores familiares distribuidos de la siguiente forma: Minya (93), Assiut (96), Sohag (151), Quena (63) y Luxor (31). Para la investigación de evaluación rápida se seleccionó una muestra aleatoria de 195 productores familiares.

Se seleccionó un coordinador responsable de la selección de la muestra, la capacitación de los entrevistadores y la revisión de los cuestionarios completados en cada una de las gobernaciones del estudio. El coordinador seleccionó a su vez diez entrevistadores por gobernación para la realización de la encuesta estructurada y se asignó un equipo adicional de dos entrevistadores para la investigación de evaluación rápida, los cuales fueron capacitados en el proceso de recolección de datos. Para la encuesta estructurada, las entrevistas con los productores se realizaron de forma individual, mientras que, para la investigación de evaluación rápida, se organizaron grupos de discusión de 5 a 6 participantes, en el caso de los productores familiares, y entrevistas individuales con los veterinarios oficiales de campo en cada una de las gobernaciones objeto del estudio. El período de recolección de datos de campo se limitó a un total de cinco días. La información obtenida en el terreno fue procesada usando el software informático SPSS ${ }^{\circledR}(\mathrm{Nie}$ et al.,1970). Los resultados de la encuesta estructurada y la investigación de evaluación rápida fueron analizados, comparados y combinados.

\section{RESULTADOS}

\section{Características de lOS enCUeStados}

El rango de edad de los productores familiares encuestados en las cinco gobernaciones objeto del estudio está comprendido entre los 17 y los 80 años, con una edad promedio de 49 años. Estos individuos poseen familias con un tamaño promedio de 7 miembros, donde se manifiesta una clara prevalencia de familias numerosas de entre 5 y 12 miembros (78,8\%). El grado de alfabetización de los entrevistados es bastante limitado, siendo que el 31,9\% de los mismos son analfabetos, el 44,0\% son capaces de leer y escribir y tan solo el $24,1 \%$ ha cursado estudios primarios y/o secundarios.

\section{TIERRA CULTIVADA Y RÉGIMEN DE PROPIEDAD}

Algo más de la mitad de los productores encuestados $(55,8 \%)$ operan en pequeñas explotaciones rurales de menos de 3 feddans (1,26 hectáreas) de superficie. Del área total de tierra cultivada, el 40,8\% de los encuestados dedican menos de un feddan para el cultivo de forraje para el ganado, lo que en promedio representa menos de un tercio de la superficie que cultivan (Figura 2). Algo más de la mitad de los encuestados (52,3\%) son propietarios de la tierra que explotan, no obstante, el resto lo hacen en régimen de arrendamiento, ya sea pagando al propietario una cantidad de dinero preestablecida $(38,5 \%)$ o un porcentaje de la cosecha $(13,8 \%)$.

\section{CRÍA DE BOVINOS Y RÉGIMEN DE PROPIEDAD}

La mayoría de los productores encuestados poseen ganado bovino local (Baladi), ya sean vacas de vientre $(76,3 \%)$, terneras y novillas $(44,0 \%)$, animales en cebo $(35,2 \%)$ o toros $(3,7 \%)$. El número de productores que cría bovinos cruzados, ya sean vacas de vientre $(43,5 \%)$, terneras y novillas $(25,1 \%)$, animales en cebo $(11,7 \%)$ o toros $(0,9 \%)$, es bastante inferior. En todas las gobernaciones, la gran mayoría de los encuestados tienen entre 1 y 3 vacas de vientre, ya sean bovinos locales $(90,0 \%)$ o cruzados $(87,6 \%)$, y 


\begin{tabular}{lcccccc}
\hline \multicolumn{7}{l}{ Tabla I. Distribución de bovinos de raza local/cruzados (Distribution of local/crossbreed cattle). } \\
\hline Gobernaciones & Minya & Assiut & Sohag & Quena & Luxor & TOTAL \\
Vacas de vientre & $51 / 30$ & $91 / 48$ & $109 / 64$ & $54 / 32$ & $26 / 15$ & $331 / 189$ \\
Terneras y novillas & $22 / 15$ & $63 / 39$ & $55 / 28$ & $33 / 17$ & $18 / 9$ & $191 / 108$ \\
Animales en cebo & $39 / 16$ & $52 / 17$ & $23 / 7$ & $33 / 9$ & $6 / 2$ & $153 / 51$ \\
Toros & $3 / 1$ & $4 / 1$ & $6 / 2$ & $2 / 0$ & $1 / 0$ & $16 / 4$ \\
\hline
\end{tabular}

entre 1 y 2 terneras y novilla, tanto locales $(90,6 \%)$ como cruzados $(90,9 \%)$ (Tabla I). Además de la especie bovina, los encuestados reportan poseer búfalos $(43,2 \%)$, pequeños rumiantes $(35,8 \%)$, équidos $(27,6 \%)$ y camellos $(7,4 \%)$. La mayor parte aseguran ser propietarios de sus animales $(87,3 \%)$, pero también hay quien comparte la propiedad de éstos $(21,6 \%)$, generalmente con parientes o amigos, y solo unos pocos crían animales que no son de su propiedad $(14,7 \%)$, generalmente animales de cebo.

\section{INSUMOS PARA LA CRÍA DE BOVINOS}

\section{ALIMENTACIÓN}

Los productores familiares dependen fundamentalmente del cultivo de forraje verde para la alimentación del ganado bovino, principalmente berseem o trébol egipcio (Trifolium alexandrinum) $(91,4 \%)$, y en menor medida sorgo forrajero (Sorghum bicolor) $(46,7 \%)$, producidos durante el invierno. Aun así, todos los productores compran alimentos para el ganado en mayor o menor medida, fundamentalmente torta de semilla de algodón $(86,6 \%)$, maíz $(57,6 \%)$,soja $(53,1 \%)$, afrecho y paja de trigo $(52,4 \%)$ y/o forraje seco henificado $(41,3 \%)$, gastando una media 600 libras egipcias (LE) mensuales $(3,5 \mathrm{LE}=1 \mathrm{US} \$)$, valor éste que aumenta durante la temporada de verano debido a la disminución en la producción de forraje verde (Tabla II).

El 94,5\% de los productores afirman que acuden al veterinario cuando sus animales están enfermos, mientras que el resto consulta con los vecinos y/o suministra remedios populares. El 53,4\% de los productores indica que el veterinario también les
Tabla II. Costo mensual en alimentación bovina (Monthly cost in cattle feeding).

\begin{tabular}{lcccc}
\hline Costo mensual & \multicolumn{2}{c}{ Período de invierno } & \multicolumn{2}{c}{ Período de verano } \\
\hline $\begin{array}{l}\text { Libras Egipcias } \\
\text { (LE) }\end{array}$ & Productores & $\%$ & Productores & $\%$ \\
\hline$<90$ & 7 & 1,6 & 8 & 1,8 \\
$90-149$ & 25 & 5,8 & 27 & 6,2 \\
$150-209$ & 53 & 7,1 & 31 & 12,2 \\
$210-269$ & 24 & 5,5 & 26 & 6 \\
$270-1.200$ & 259 & 59,7 & 262 & 60,4 \\
$>1.200$ & 66 & 15,2 & 80 & 18,4 \\
\hline TOTAL & 434 & 100 & 434 & 100 \\
\hline
\end{tabular}

visita en sus explotaciones, ya sea para atender animales enfermos $(43,8 \%)$, vacunar $(40,8 \%)$, realizar inseminación artificial $(18,2 \%)$, chequeos de rutina $(13,8 \%)$ y / o para proporcionar servicios de extensión pecuaria $(11,1 \%)$. El productor promedio gasta unas $300 \mathrm{LE}$ anualmente en servicios veterinarios para su ganado.

\section{REPRODUCCIÓN}

La mayoría de los productores $(85,5 \%)$ menciona tener conocimiento del uso de la inseminación artificial como técnica reproductiva para el ganado bovino, les han informado el veterinario $(66,6 \%)$ o sus familiares y amigos (18,9\%), sin embargo, la mitad de ellos (43,5\%) nunca la ha utilizado en sus vacas. De los productores que conocen y han utilizado la inseminación artificial (42,0\%), el 27,4\% admite recurrir a ella solo cuando la monta natural no funciona. El 85,8\% de los producto-

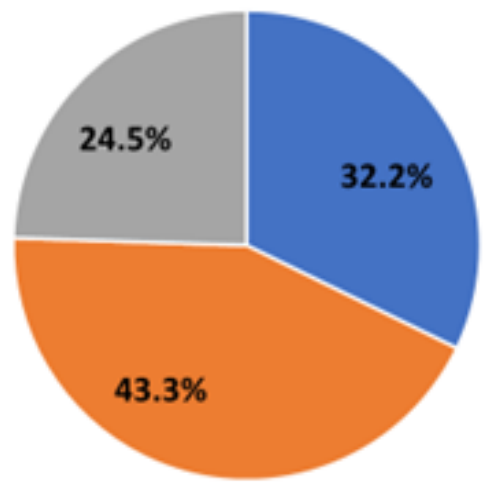

Vacas

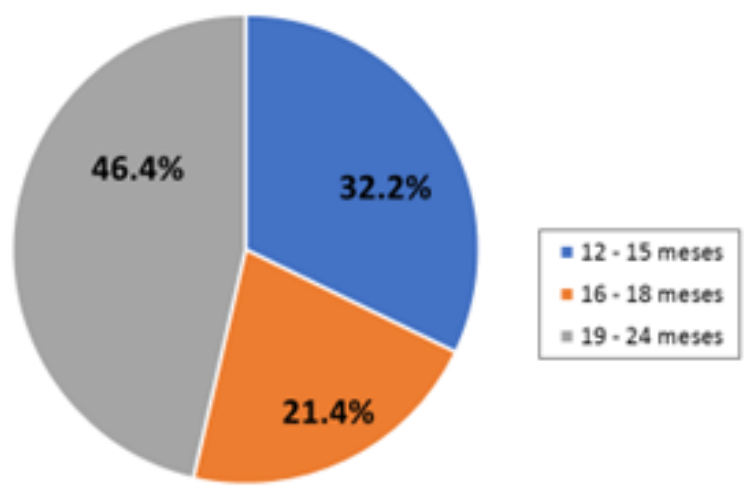

Toros

Figura 3. Edad de la primera cubrición/monta en el ganado bovino (Age at first mating in cattle). 
res que usan esta técnica reproductivapagan entre 3 y 10 LE por el servicio que gestiona el sector público, mientras que si acuden al sector privado pagan entre 6 y 20 LE. El costo del desplazamiento del veterinario es la razón principal de la discrepancia de precios, con un promedio de 5,4 LE en el caso del sector público y de $11 \mathrm{LE}$ en el caso del sector privado. El costo de la monta natural y el de la inseminación artificial en un punto fijo público son bastante similares.

\section{PRODUCTIVIDAD DE LA CRÍA DE BOVINOS}

\section{PRODUCCIÓN Y VENTA DE LECHE}

La producción diaria promedio de leche de la vaca local o Baladi es de 4,6 kg en invierno y de 4,1 kg en verano, con un rendimiento máximo promedio de 5,4 $\mathrm{kg}$ por día. En la vaca cruzada es de 7,6 kg en invierno y $5,6 \mathrm{~kg}$ en verano, con un rendimiento de $8,9 \mathrm{~kg}$. En las vacas de raza exótica es de $10,3 \mathrm{~kg}$ en el invierno y $8,4 \mathrm{~kg}$ en el verano, con un rendimiento de $12,2 \mathrm{~kg}$. Así pues, la ganancia estacional entre el invierno y el verano en la producción diaria de leche es de $0,5 \mathrm{~kg}$ para la vaca local o Baladi, de $2,0 \mathrm{~kg}$ para la vaca cruzada y de $1,9 \mathrm{~kg}$ para la vaca de raza exótica. Los productores indican que la leche producida es consumida por la familia $(91,9 \%)$ y tan solo unos pocos la venden $(8,1 \%)$, ya sea fresca o después de procesarla en el hogar. Sobre la utilización futura de una posible producción de leche adicional, el 83,9\% de los encuestados continúa afirmando que sería consumiría por la familia, mientras que un $16,1 \%$ indica que vendería los excedentes. El precio promedio de un kilogramo de leche fresca es de 1,31 LE en invierno y 1,25 LE en verano, encontrándose el precio más alto en la gobernación de Luxor y el más bajo en Minya.

\section{PRODUCCIÓN Y VENTA DE CARNE}

El 83,6\% de los productores indica que ceba de 1 a 3 cabezas de ganado bovino, tanto de raza local o Baladi como cruzado. Alrededor del 77,3\% de los productores venden sus bovinos de cebo con un peso inferior a 267 $\mathrm{kg}$. Aproximadamente la mitad de los productores $(53,9 \%)$ venden sus animales en cebo con una edad comprendida entre los 6 y 12 meses, mientras que el resto los venden entre 12 y 24 meses $(33,8 \%)$ o más de 24 meses $(12,2 \%)$. El precio promedio de bovinos en cebo, si se venden con menos de 12 meses, es de 1.051 LE, de 1.983 LE con entre 12 y 24 meses y de 2.978 LE con más de 24 meses. Así pues, el precio promedio por kilogramo de peso vivo de bovinos en cebo en las cuatro gobernaciones del estudio es de 7,5 LE.

\section{Otros ASPECTOS RELACIONADOS CON LA CRÍA DE BOVINOS}

CONOCIMIENTOS, APTITUDES Y PRÁCTICAS QUE INFLUYEN EN LA CRÍA DE BOVINOS

El $72,7 \%$ de los productores afirma no mantener registros individuales de alimentación (ej.: cantidad, origen, precio de compra, etc.), producción (ej.: leche, incremento de peso, valor de venta, etc.), tratamientos o visitas del veterinario, reproducción (ej.: fechas de montas naturales o inseminaciones artificiales, partos, etc.), $\mathrm{u}$ otros relacionados con la cría de bovinos.

La mayoría de los productores $(96,2 \%)$ reconocen como signos del celo en sus vacas los cambios en el comportamiento y la presencia de moco vaginal. Después de detectar los primeros signos del celo, el 73,2\% de los productores realiza la cubrición entre las 6 y 12 horas siguientes, el 18,6\% entre las 4 y 5 horas y el 8,2\% entre 1 y 3 horas. El 32,2\% de los productores cubren sus vacas por primera vez a la edad de 12-15 meses, el $43,3 \%$ a los $16-18$ meses y el $24,5 \%$ a los $19-24$ meses. En cuanto a los toros, la mayoría de los productores informan que la primera monta la realizan a los 18 meses $(21,4 \%)$ o a los 24 meses (46,4\%) (Figura 3 ).

La mayoría de los productores (70,9\%) que conocen la inseminación artificial prefiere utilizar la monta natural. Las razones expuestas son el insuficiente conocimiento de la técnica $(4,7 \%)$ o el no estar convencidos de sus beneficios (36,2\%). Otros indican la indisponibilidad $(18,6 \%)$ o la lejanía $(11,8 \%)$ de este servicio en sus municipios, comparado con el acceso más favorable a toros para monta natural $(13,3 \%)$ y su menor costo $(1,1 \%)$. Algunos creen que es mejor usar la monta natural en el primer celo de la vaca $(6,8 \%)$, también hay quien considera que la técnica no es compatible con sus creencias religiosas $(6,1 \%)$ o les preocupa que pueda afectar a sus animales $(1,4 \%)$, transmitiéndoles enfermedades o imposibilitándolos posteriormente para la monta natural.

Para corroborar estas respuestas, se les preguntó a los productores que conocen y nunca han usado la inseminación artificial (43,5\%), en qué condiciones la utilizarían. Los requisitos exigidos fueron que el servicio estuviera disponible en todo momento (26,8\%), cerca de sus explotaciones $(29,3 \%)$ o a domicilio $(7,3 \%)$, así como que les garanticen la calidad del semen utilizado $(11,0 \%)$ y la fiabilidad de la técnica $(19,5 \%)$. Otros aseguran que solo la utilizarían si fuera más económica que la monta natural $(2,4 \%)$ o cuando ésta última no funcionase $(3,7 \%)$. Solo el $15,2 \%$ de los productores expresó que no usarían esta técnica bajo ninguna circunstancia.

\section{INTERÉS POR RAZAS BOVINAS EXÓTICAS}

Cuando se preguntó a los productores familiares si estarían interesados en adquirir razas bovinas exóticas, el 45,2\% afirmó no estarlo, indicando diferentes razones, tales como el alto costo para su adquisición $(33,8 \%)$ y alimentación $(28,6 \%)$, su mayor susceptibilidad a las enfermedades $(25,0 \%)$ o la dificultad para vender la mayor cantidad de leche y carne que éstas producen $(12,7 \%)$. Los productores interesados en este tipo de animales $(54,8 \%)$, preferirían adquirir la Frisona $(64,4 \%)$, Parda Alpina (22,2\%), Tarantaise $(9,6 \%)$ y/o Abondance $(3,5 \%)$, debido a su mayor producción de leche (Frisona) y carne (Parda Alpina), su adaptabilidad a las condiciones locales (Frisona y Parda Alpina), su capa parecida a la de las vacas locales (Parda Alpina y Tarantaise ), su facilidad al parto (Parda Alpina), su larga historia en el país (Frisona) o porque se las han recomendado vecinos y/o familiares (todas).

\section{CRÉDITOS Y PRÉSTAMOS PARA LA PRODUCCIÓN PECUARIA}

El 88,7\% de los productores expresan voluntad por aumentar su producción de leche y/o carne a través de una mejor alimentación del ganado o de la adquisición de mejores animales, pero el $60,0 \%$ indica que necesita- 
rían créditos o préstamos para ello. Aunque algo más de la mitad de estos últimos $(53,0 \%)$ los ha conseguido alguna vez, de entre los productores que aseguran nunca haberlos obtenido, el 41,3\% argumenta que no los consiguen porque no pueden garantizar su pago. Los productores que han obtenido alguna vez préstamos o créditos mencionan haberlos recibido del banco $(45,0 \%)$, de familiares y/o amigos $(27,2 \%)$, de vecinos $(16,0 \%)$ y de los comerciantes de su localidad (11,8\%).

\section{DISCUSIÓN}

La muestra seleccionada se encuadra bastante con lo descrito por la literatura (CAPMAS 2017) con relación a las características de la población rural del Alto Egipto, pues la mayoría de los productores encuestados poseen familias numerosas y el nivel de alfabetización de éstos es bastante bajo. Además, y considerando que la mitad de ellos no son propietarios de la tierra, se podría considerar también que una buena proporción de los encuestados se encuentra en situación de pobreza. La relativamente alta edad promedio de los productores (49 años) no solo refleja una sociedad rural de índole patriarcal, donde diferentes generaciones conviven bajo el mismo techo y trabajan la misma tierra, también muestra las dificultades que enfrentan los jóvenes productores rurales para establecerse de forma independiente, debido a la escasez y el limitado acceso a la tierra en la región, lo cual fomenta el éxodo rural hacia las grandes ciudades egipcias y otros países de la región (Bush 2016).

De acuerdo nuevamente con lo que describe la bibliografía (ENID 2011, Thomsonet al.,2000; Lewis 2008), es posible afirmar que la gran mayoría de los encuestados podría clasificarse como productores familiares, dentro de la categoría de extensivos con la que se han diferenciado los sistemas de producción pecuaria en Egipto. Mas de la mitad de los productores encuestados explota terrenos con menos de 3 feddans de superficie, de los que cerca de un tercio es dedicada al cultivo de forrajes. Son propietarios de un promedio de 3 cabezas de ganado, mayoritariamente vacas de vientre de raza local (Baladi), que crían junto a otras especies animales en la misma explotación. Esto sucede por igual en las cinco gobernaciones muestreadas en el Alto Egipto, lo cual revela la gran atomización del sector pecuario en la región, como también fue indicado en la literatura consultada (FAO 2017, MALR 2006,Thomson et al.,2000).

La alimentación representa el insumo más costoso para la cría de bovinos y supone la principal limitación para la expansión de la producción ganadera en el Alto Egipto. La mayoría de los productores dependen del berseem o trébol egipcio para la alimentación animal, sobre todo en la temporada de invierno, utilizando también otros productos para completar la alimentación del ganado, principalmente durante el verano. Algunos de ellos pueden ser parcialmente producidos en la granja, otros se compran fuera de la explotación. El maíz y la soja, principales ingredientes de los concentrados animales, son importados en su mayor parte y su precio se ve afectado por la disminución del valor de la libra egipcia (LE) frente a las divisas extranjeras, encareciendo el costo de la alimentación animal. Debido a que no pueden permitirse alimentar una gran cantidad de animales, los productores familiares se concentran en las vacas de vientre y venden los terneros después de un breve periodo de ceba.

En general, los servicios veterinarios en el Alto Egipto son bastante accesibles para los productores familiares. En su mayor parte son de carácter público y están enfocados principalmente a cubrir las necesidades básicas en materia de salud animal del sector, por lo que tampoco suponen un gasto excesivo para el ganadero, estimándose en unas 100 LE anuales por animal. La mayor parte de los veterinarios oficiales trabajan en horario de mañana para el sector público y en la tarde como veterinarios privados, pero no dedican suficiente tiempo a actividades de extensión y capacitación. De hecho, muy pocos productores afirman recibir este tipo de servicio, los cuales son muy necesarios en el Alto Egipto, como se aprecia cuando se analizan los conocimientos, aptitudes y prácticas de los productores familiares frente a aspectos básicos de gestión de su empresa pecuaria (ej.: ausencia de registros, prácticas reproductivas erróneas, desconocimiento y/o rechazo de nuevas tecnologías, etc.).

La reproducción es el insumo para la cría de bovinos más económico de los evaluados en este estudio. El costo de la inseminación artificial ofertada por los servicios veterinarios oficiales es equiparable al costo de la monta natural, pero si el productor acude al sector privado el precio es prácticamente el doble. Para ahorrarse los costos por desplazamiento del veterinario, los productores acostumbran a acudir con sus animales a los puntos fijos de inseminación artificial. Estos puntos fijos están localizados en las unidades clínicas veterinarias establecidas por el gobierno en las localidades rurales del Alto Egipto, por lo general el servicio es público en la mañana y en la tarde pasa a ser privado. No obstante, este servicio no se encuentra disponible en todos los municipios encuestados en la región, comparado con el acceso más favorable a toros para monta natural, y existe un cierto desconocimiento, e incluso perjuicio, con respecto al uso de esta técnica reproductiva.

La productividad de la cría de bovinos en el Alto Egipto sigue un patrón estacional y se ve fundamentalmente limitada por la alimentación, que generalmente es más abundante y de mayor calidad en el invierno. Aunque las razas exóticas producen hasta 2,3 veces más leche que las locales y 1,4 más veces que las cruzadas, en el verano la producción de las primeras disminuye en un $18,4 \%$, mientras que en las vacas locales se reduce un $10,9 \%$ y en las cruzadas un $26,3 \%$. Además, evaluando las razones de los productores familiares para no adquirir razas bovinas exóticas, se podría presumir que los propietarios de estas razas proporcionalmente invierten más en la alimentación de sus animales que aquellos que poseen animales locales o cruzados, lo cual podría explicar la gran disminución de la productividad de las vacas cruzadas en el verano, que son alimentadas de igual forma que las razas locales. Esto refleja la importancia de la nutrición para las diferentes razas en relación con los rendimientos en la producción de leche. 
En lugar de vender la leche, la mayoría de los productores prefieren que sea consumida en el núcleo familiar, ya sea fresca o en forma procesada, y afirman que, incluso si pudieran producir más cantidad en el futuro continuarían sin venderla, lo cual concuerda con lo descrito en la bibliografía (Radwan 2016, Soliman \& Mashhour 2011), algo muy distinto a lo que courre en otros sistemas agroecológios (Vásquez-Jaramillo et al.,2018). Sin embargo, la mayoría de los productores expresan voluntad por aumentar su producción de leche y/o carne a través de una mejor alimentación del ganado o de la adquisición de mejores animales y, algo más de la mitad, muestra interés en adquirir razas bovinas exóticas, principalmente la Frisona, debido a su mayor producción de leche. Así pues, existe una duda razonable con relación a la sinceridad de las respuestas cuando los encuestados aseguran que no comercializan leche y/o productos lácteos. Curiosamente, en términos generales los precios de la leche fresca son más bajos en el verano, época del año en el que la producción es menor, y la diferencia de precios entre gobernaciones es bastante notable. En general se aprecia un deseo de incorporar nuevas tecnologías de apoyo al comercio (Elghannam et al.,2018)

Algo más de la mitad de los productores familiares encuestados poseen animales en cebo, pero raramente superan las 3 cabezas, que generalmente son animales machos de raza local o Baladi. Para maximizar sus ganancias, los productores familiares encuentran más atractivo vender sus animales en cebo antes de cumplir los 12 meses de vida y con un peso medio de 140 $\mathrm{kg}$. Generalmente, vender animales en cebo con 12-24 meses y un peso medio de $260 \mathrm{~kg}$, o con más de 2 años y un peso medio de $400 \mathrm{~kg}$, no resulta rentable al productor familiar, debido al costo de la alimentación, que aumenta con la edad de los animales, y a su gran necesidad de dinero rápido en efectivo. Esto entra claramente en conflicto con la creciente demanda interna de carne roja en el país y con los objetivos del gobierno egipcio de reducir las importaciones de carne al mínimo (FAO 2017, MALR 2009).

Se ha dedicado especial atención a los conocimientos, aptitudes y prácticas de los encuestados con relación al mejoramiento bovino para aumentar la producción de leche y carne, mediante el uso de razas exóticas y de la inseminación artificial, objetivos principales del proyecto hispano-egipcio a través del cual se realizó este estudio. Los productores que muestran interés por adquirir razas exóticas mencionan las cuatro que conocen, introducidas de forma escalonada en la región, así pues, sus respuestas no son particularmente significativas. Sin embargo, se percibe una cierta preferencia por razas rústicas, capas similares al ganado autóctono y mayor adaptabilidad a las condiciones locales. La mayoría de los productores que usan la inseminación artificial se encuentran a menos de $2,5 \mathrm{~km}$ de distancia de un punto fijo, siendo más favorables a su uso cuanto más jóvenes son. El costo de la inseminación artificial no es una consideración importante, de hecho, la mayoría de los productores están dispuestos a pagar más por un servicio privado a cambio de garantías de calidad y una mejor conveniencia para su uso.
El alto costo de adquisición y manutención es la razón principal por la cual casi la mitad de los productores pecuarios entrevistados no desean adquirir razas bovinas exóticas. Estos productores entienden que, en comparación con las razas autóctonas, estos animales tienen mayores requisitos nutricionales y son más susceptibles a las enfermedades. Además, unos pocos consideran que el mercado local no puede asumir la mayor producción que los animales de razas exóticas proporcionarían. Esto bien podría ser cierto para la leche, debido al supuesto impedimento socio-cultural para vender este producto. Sin embargo, no es el caso de la carne pues, en comparación con otros tipos de carne importada, la carne bovina de producción nacional es bastante apreciada, alcanzando precios más altos en el mercado, representando una buena fuente de ingresos para la agricultura familiar egipcia (Osman et al., 2016).

La principal razón por la que los productores no usan la inseminación artificial está relacionada con la disponibilidad del servicio. Algunos argumentan que no hay puntos fijos públicos en sus municipios, están lejos de sus granjas o que el servicio no está siempre disponible. Esto último puede deberse a la falta de dosis seminales, nitrógeno líquido, equipos y/o suministros, a la indisponibilidad del veterinario o a la inaccesibilidad a los puntos fijos después del horario oficial de trabajo. El desconocimiento o la falta de confianza en la inseminación artificial es el siguiente factor más importante y podría deberse, en parte, a un servicio de extensión pecuaria insuficiente y/o ineficiente, pero también hay quejas sobre la calidad de las dosis seminales de producción nacional. Por otro lado, existen una tendencia a usar la inseminación artificial cuando la monta natural no funciona, siendo probable que en esos casos existan problemas de infertilidad en el ganado, lo que provoca la pérdida de confianza del productor.

\section{CONCLUSIONES}

El estudio destacó como los principales obstáculos a los que se enfrentan las comunidades rurales del Alto Egipto dedicadas a la cría de bovinos bajo sistemas tradicionales de agricultura familiar, son el limitado acceso a la tierra, el relativo alto costo y escasa disponibilidad de insumos y servicios básicos, la marcada estacionalidad de la productividad animal y la dificultad para acceder a créditos y préstamos agropecuarios.

Resultó también evidente que el sector público veterinario tiene dificultades para proporcionar un adecuado servicio de extensión pecuaria, que cubra las necesidades básicas de capacitación de los productores familiares identificadas a través de este estudio, lo cual es un elemento muy importante para el desarrollo del sector ganadero nacional.

La dificultad para comercializar la leche y carne es también un gran obstáculo para los productores familiares de la región y una barrera que los mantiene en los límites de la pobreza. Una transformación institucional que pueda defender los intereses de los productores familiares contra los posibles monopolios en el sector del agronegocio y ayude a abordar sus limitaciones 
de comercialización, contribuiría al desarrollo de la agricultura familiar y particularmente, de la cría de bovinos en el Alto Egipto.

Además, las estrategias de mejora genética nacionales han descuidado las razas bovinas autóctonas (Baladi), amenazadas por los programas de cruzamiento y las políticas de desarrollo económico, a pesar de ser consideradas por los productores como las más adaptadas y resistentes a las severas condiciones locales, permitiendo una mejor transformación de los limitados recursos forrajeros producidos en las pequeñas explotaciones familiares de la región.

A nivel nacional, estos hallazgos plantean muchas cuestiones para los responsables políticos, las organizaciones de cooperación y la sociedad en general, con respecto a la situación de la agricultura familiar. Todas las estrategias de intervención con vistas a incrementar las fuentes de ingresos y aliviar la pobreza en las comunidades rurales del sur de Egipto, así como preservar y administrar los recursos genéticos que explotan a nivel comunitario, deben tener en cuenta todas estas circunstancias particulares.

\section{AGRADECIMIENTOS}

Los autores desean agradecer a la Agencia Española de Cooperación Internacional para el Desarrollo (AECID), que financió este estudio a través del "Proyecto hispanoegipcio para el desarrollo de la inseminación artificial en el Alto Egipto". Un agradecimiento muy especial también a todo el personal y consultores del proyecto, a la contraparte nacional de los servicios veterinarios y a los productores familiares egipcios, que colaboraron en la planificación y en la ejecución a nivel de campo.

\section{BIBLIOGRAFÍA}

Alary V, Aboul-Naga A, El Shafie M, Abdelkrim N, Hamdon H \& Metawi $\mathrm{H}$ 2015. Roles of small ruminants in rural livelihood improvement Comparative analysis in Egypt. Revue d'élevage et de médecine vétérinaire des pays tropicaux, vol. 68 no. 2-3, pp. 79-85.

Bush R 2016. Family farming in the Near East and North Africa. Working Paper No. 151. International Policy Centre for Inclusive Growth. Published by FAO \& UNDP. http://www.fao.org/3/a-i6537e.pdf

CAPMAS 2017. Population and Education Statistics. Central Agency for Public Mobilization and Statistics. Cairo, Egypt. http://www. msrintranet.capmas.gov.eg/

CIA 2018. Africa: Egypt. The World Factbook. Central Intelligence Agency. Washington DC, USA https://www.cia.gov/library/publications/ the-world-factbook/geos/eg.html

Coghlan D \& Brydon-Miller M 2014. Participatory Rapid Appraisal. In: The SAGE Encyclopedia of Action Research. http://dx.doi. org/10.4135/9781446294406.n267

ECES 2017. Egypt's Economic Profile and Statistics. The Egyptian Center for Economic Studies. http://www.eces.org.eg/MediaFiles/ Uploaded_Files/e7bOad3f.pdf

El-Ramady HR, El-Marsafawy SM \& Lewis LN 2013. Sustainable Agriculture and Climate Changes in Egypt. Sustainable Agriculture Reviews, vol 12. pp 41-95.

El-Shimi S \& Arafa S 2011. Biogas technology transfer to rural communities in Egypt. Agricultural Research Center and The American University in Cairo, Cairo, Egypt. https://web.archive.org/ web/20111123011949/http://www.egyptchm.org/chm/implementation/pdf/tech_transfer/EL_S0291.pdf
Elghannam A and Mesías FJ 2018. Social networks as a new marketing channel for animal food products: a qualitative study in Spain. Archivos de Zootecnia, vol. 67, no. 258, pp. 260-268.

ENID 2011. Towards More Efficient Use of Agricultural Resources in Upper Egypt: Optimum Cropping Pattern Under Different Scenarios in Qena (Policy Brief 00 1). EgyptNetwork for Integrated Development. http://www.enid.org.eg/Uploads/PDF/PB1_cropping_pattern.pdf

FAO 2005. Fertilizer use by crop in Egypt. Rome, viewed 11 October 2018.www.fao.org/docrep/008/y5863e/y5863e00.htm

FAO 2017. Country Brief: Egypt. Africa Sustainable Livestock 2050. Rome, viewed 14 November 2018. http://www.fao.org/3/a-i7312e.pdf

FAO 2018. Livestock production systems spotlight: Egypt. Cattle and buffaloes and poultry sectors. Africa Sustainable Livestock 2050. Rome, viewed 15November 2018. http://www.fao.org/3/i8477en/ I8477EN.pdf

FAOSTAT 2018. Indicadores por País - Egipto. Rome, viewed 10 October 2018. http://www.fao.org/faostat/es/\#country/59

Fitch J \& Soliman I 1981. The Livestock Economy in Egypt: an appraisal of the current situation. Economic Working Paper Series No. 29, University of California. Davis, USA, 47 pp. http://ageconsearch.umn. edu/record/232850/files/ucdavis-egyptproject-029.pdf

Galal S 2007. Farm animal genetic resources in Egypt: factsheet. Egyptian Journal of Animal Production, vol. 44, no. 1, pp. 1-23.

IFAD 2015. Arab Republic of Egypt. Country strategic opportunities programme. Rome, viewed 20 October 2018. https://www.ifad. org/documents/3871 1644/40050262/02_07_15\%20Egypt\%20 COSOP_0018-25143096-1630_8579.pdf/b083a4e5-af5b-4ff89842-e99731 aa3317? 1517983626097

Lewis LN 2008. 'Egypt's Futures Depends on Agriculture and Wisdom'. Agricultural Issues Center. University of California. Davis, USA, 360 pp. http://aic.ucdavis.edu/calmed/August $\% 2008, \% 20$ final $\% 20$ 2 d\%20Egypt.pdf

Lewis-Beck MS, Bryman A \& Liao TF 2004. Structured Interview. In: The SAGEEncyclopedia of Social Science Research Method. Ed. SAGE, Thousand Oaks, USA. http://dx.doi.org/10.4135/9781412950589. n909

MAEC 2018. Ficha país: República Árabe de Egipto. Oficina de Información Diplomática. Ministerio de Asuntos Exteriores y Cooperación, Madrid, España. http://www.exteriores.gob.es/Documents/ FichasPais/EGIPTO_FICHA\%2OPAIS.pdf

MALR 2006. Animal Production Sector Databases. Ministry of Agriculture and Land Reclamation. Cairo, Egypt. http://www.agri.gov.eg

MALR 2009. Sustainable Agricultural Development Strategy towards 2030. Agricultural Research \& Development Council. Ministry of Agriculture and Land Reclamation. Cairo, Egypt. http://far-malr.gov. eg/pdf/en/Full\%20SADS2030.pdf

Nie NH, Bent DH \& Hull CH 1970. SPSS: statistical package for the social sciences. Ed. McGraw-Hill. 343 p. https://books.google.es/ books? $i d=$ KKFXAAAAMAA

Osman MA, Alary V, Khalil M, Elbeltagy A\& Tourrand J-F, Moulin C-H 2016. Adaptability and suitability of local cattle breeds in Egypt: Farmers and actors' perceptions and practices of the cattle value chain. Revue d'élevage et de médecine vétérinaire des pays tropicaux, vol. 69 no. 3, pp. 95-103.

Radwan MAA 2016. 'Characterization of milk and veal production chains of buffalo under crop livestock production system in Egypt'. PhD Thesis, Cairo University, Cairo, Egypt.

Rangel-Quintos J, de Pablos-Heredero C, Vélez A, Espinosa JA, Perea J, Angón E y García, A 2016 Sustentabilidad social de agroecosistemas bovinos de doble propósito en México. Archivos de Zootecnia. vol. 65 no. 251 , pp.315-319.

Soliman I \& Mashhour A 2011. Dairy Marketing System Performance in Egypt. MPRA Paper 66799, University Library of Munich, Germany. https://mpra.ub.uni-muenchen.de/66799/8/MPRA_paper_66799.pdf

Tabana A, Van Keulen H, Tamminga S \& Gomaa I 2000. 'Animal production systems in Egypt: their roles, classification, description and 
potential contribution to development'. Proceedings of the $3^{\text {rd }}$ All Africa Conference on Animal Agriculture \& $11^{\text {th }}$ Conference of the Egyptian Society of Animal Production. Alexandria, Egypt, p. 635-642. Thomson EF, von Kaufmann R, Li Pun H, Treacher T \& van Houten H. (eds). 2000. 'Global Agenda for Livestock Research'. Proceedings of a Consultation on Setting Livestock Research Priorities in West Asia and North Africa (WANA) Region. Aleppo, Syria, 172 pp.

Torres Y, Pablos-Heredero C de, Morantes M, Rangel J, Espinosa JA, Angón E y García A 2016. El papel de la mujer en la explotación de ganado bovino de doble propósito en Ecuador. Archivos de Zootecnia. vol. 65 no. 251, pp. 309-314.

Vásquez-Jaramillo C, Barrios D y Cerón-Muñoz MF 2018 Estudio exploratorio de la calidad de vida en el trabajo de ordeñadores de sistemas de producción de leche. Archivos de Zootecnia. vol. 67 no. 258, pp. 228-233.

WBG 2018. Egypt, Arab Republic. World Bank Group. Washington DC, viewed 20 December 2018. https://datos.bancomundial.org/ pais/egipto-republica-arabe-de 